in their cohort of elderly women and highlight a need for further studies involving different populations and longer follow-up times.

Original article Diem SJ et al. (2007) Use of antidepressants and rates of hip bone loss in older women. Arch Intern Med 167: $1240-1245$

\section{Detection of ANCA with several assays in patients with Wegener's granulomatosis}

The role of antineutrophilic cytoplasmic antibody (ANCA) in the pathogenicity of Wegener's granulomatosis is controversial, and the variability in results obtained from different serum ANCA assays is considerable. Finkielman and colleagues used several assays to determine the frequency of ANCA presence in a cohort of patients with Wegener's granulomatosis, and examined whether disease severity affects the results of these assays.

Serum samples were obtained from 180 patients (mean age 47 years, 60\% men, 92\% white, median time since symptom onset 17 months) who were enrolled in the Wegener's Granulomatosis Etanercept Trial. Of these, 128 (71\%) had severe disease (defined as lifethreatening or vital-organ-threatening) and 52 (29\%) had limited disease. Baseline serum samples were assayed for several ANCA subtypes using standard indirect immunofluorescence, direct ELISA, and two capture ELISA assays.

Immunofluorescence detected ANCA in 160 (89\%) patients (cytoplasmic ANCA staining [C-ANCA] in 136 patients, perinuclear ANCA staining [p-ANCA] in 24 patients). Direct ELISA detected proteinase 3 (PR3)-ANCA in 133 (74\%) patients; this ANCA subtype was detected in 136 (76\%) patients and 148 (82\%) patients using the mature-PR3 capture ELISA and anti-c-myc capture ELISA, respectively. Overall, 166 (92\%) patients had detectable ANCA. Patients with severe disease were more likely to be ANCA-positive than those with limited disease (96\% vs 83\%).

The authors conclude that ANCA is detectable in almost all patients with Wegener's granulomatosis, and that use of the full range of assays is required to characterize the presence of all PR3-ANCA accurately and reliably.

Original article Finkielman JD et al. (2007) ANCA are detectable in nearly all patients with active severe Wegener's granulomatosis. Am J Med 120: 643.e9-e14

\section{Twice-weekly laser therapy does not improve effects of stretching in patients with FMS}

Fibromyalgia syndrome (FMS) is characterized by both widespread pain and tender points sensitive to touch. Muscle-stretching exercises reduce symptoms of FMS; however, patients often show a low pain threshold at tender points. Several studies have reported analgesic properties of low-dose laser. In this study, Matsutani et al. evaluate the effectiveness of low-dose laser therapy, in concert with muscle-stretching exercises, for improving the quality of life of patients with FMS.

Twenty female patients, randomly distributed to either a stretching group (mean age 44 years; $n=10$ ) or a laser therapy plus stretching group (mean age 45 years; $n=10$ ), were evaluated in terms of pain felt, pain threshold and quality of life, before and after their treatment regime. Five muscle-stretching exercises were each performed ten times on a daily basis. The laser therapy plus stretching group also underwent twice-weekly low-dose laser application $\left(280 \mathrm{~nm}, 3 \mathrm{~J} / \mathrm{cm}^{2}\right.$, continuous mode) at each tender point before the stretching exercises were performed on those days.

Both groups experienced significantly less pain, a significant improvement in pain threshold and a significant improvement in quality of life following the five-week treatment regime. There was no difference between the two groups, however, for any of the outcomes measured.

The authors conclude that, although musclestretching exercises were beneficial for the patients involved in their study, the laser therapy did not provide any additional benefits to these patients with FMS.

Original article Matsutani LA et al. (2007) Effectiveness of muscle stretching exercises with and without laser therapy at tender points for patients with fibromyalgia. Clin Exp Rheumatol 25: 410-415

\section{Stopping anti-TNF therapy before surgery might not reduce postoperative complication rates}

Treatment with tumor necrosis factor (TNF) inhibitors is associated with an increased risk of infection; however, the safety of continuing anti-TNF therapy during the period running up to surgery is unknown. Ruyssen-Witrand and 